\title{
Miniature Poodle
}

National Cancer Institute

\section{Source}

National Cancer Institute. Miniature Poodle. NCI Thesaurus. Code C53859.

The Miniature Poodle is a medium-sized poodle with a height of 11-15 inches and weighing 15-17 pounds. 